\title{
Generalized Metropolis dynamics with a generalized master equation: An approach for time-independent and time-dependent Monte Carlo simulations of generalized spin systems
}

\author{
Roberto da Silva* \\ Instituto de Fisica, Universidade Federal do Rio Grande do Sul, Avenida Bento Gonçalves, 9500 CEP 91501-970, \\ Porto Alegre, Rio Grande do Sul, Brazil \\ José Roberto Drugowich de Felício ${ }^{\dagger}$ \\ Faculdade de Filosofia, Ciências e Letras de Ribeirão Preto, Universidade de São Paulo, Avenida Bandeirantes, \\ 3900 CEP 14040-901, Ribeirão Preto, São Paulo, Brazil \\ Alexandre Souto Martinez $z^{\ddagger}$ \\ Faculdade de Filosofia, Ciências e Letras de Ribeirão Preto, Universidade de São Paulo, Avenida Bandeirantes, \\ 3900 CEP 14040-901, Ribeirão Preto, São Paulo, Brazil and \\ Instituto Nacional de Ciência e Tecnologia em Sistemas Complexos, Rio de Janeiro, Brazil
}

(Received 17 January 2012; published 14 June 2012)

\begin{abstract}
The extension of Boltzmann-Gibbs thermostatistics, proposed by Tsallis, introduces an additional parameter $q$ to the inverse temperature $\beta$. Here, we show that a previously introduced generalized Metropolis dynamics to evolve spin models is not local and does not obey the detailed energy balance. In this dynamics, locality is only retrieved for $q=1$, which corresponds to the standard Metropolis algorithm. Nonlocality implies very time-consuming computer calculations, since the energy of the whole system must be reevaluated when a single spin is flipped. To circumvent this costly calculation, we propose a generalized master equation, which gives rise to a local generalized Metropolis dynamics that obeys the detailed energy balance. To compare the different critical values obtained with other generalized dynamics, we perform Monte Carlo simulations in equilibrium for the Ising model. By using short-time nonequilibrium numerical simulations, we also calculate for this model the critical temperature and the static and dynamical critical exponents as functions of $q$. Even for $q \neq 1$, we show that suitable time-evolving power laws can be found for each initial condition. Our numerical experiments corroborate the literature results when we use nonlocal dynamics, showing that short-time parameter determination works also in this case. However, the dynamics governed by the new master equation leads to different results for critical temperatures and also the critical exponents affecting universality classes. We further propose a simple algorithm to optimize modeling the time evolution with a power law, considering in a log-log plot two successive refinements.
\end{abstract}

DOI: 10.1103/PhysRevE.85.066707

PACS number(s): 05.10.Ln, 05.70.Ln, 02.70.Uu

\section{INTRODUCTION}

The study of the critical properties of magnetic systems plays an important role in statistical mechanics, and as a consequence also in thermodynamics. For equilibrium, the extensitivity of the entropy is a question of principle for most physicists. Nevertheless, an important issue may be raised. Many physicists believe that statistical mechanics generalizations with an extra parameter $q$ [1] are suitable for studying the optimization combinatorial process, as, for example, simulated annealing (see, e.g., Refs. [2,3]) or areas such as econophysics [4,5], population dynamics and growth models [6-9], bibliometry [10], and others.

In this paper, we generate the critical dynamics of Ising systems using a new master equation. This master equation leads to a generalized Metropolis prescription, which depends only on the spin interaction energy variations with respect to its neighborhood. Furthermore, it satisfies the detailed energy balance condition and it converges asymptotically to

\footnotetext{
*rdasilva@if.ufgrs.br

†drugo@usp.br

‡asmartinez@ffclrp.usp.br
}

the generalized Boltzmann-Gibbs weights. In Refs. [11,12] generalized prescriptions have been treated as local. Here, we demonstrate that they are instead nonlocal. However, a nonlocal prescription such as the one of Ref. [13] is numerically more expensive and destroys the phase transition. Another possibility is to recover locality. Using a special deformation of the master equation, we show how to recover locality for a generalized prescription and additionally recover the detailed energy balance in equilibrium spin systems, maintaining the system phase transition.

To apply our Metropolis prescription, we have simulated a two-dimensional Ising system in two different ways: using equilibrium Monte Carlo (MC) simulations we estimate critical temperatures for different $q$ values, and we perform timedependent simulations. In the second part, we also calculate the critical exponents corresponding to each critical temperature. Finally, we have developed an alternative methodology to refine the determination of the critical temperature. Our approach is based on the optimization of the magnetization power laws on a logarithmic scale via maximization of the determination coefficient $(r)$ of the linear fits.

Our presentation is organized as follows. In Sec. II, we briefly review the results of the critical dynamics for spin 
systems. In this review, we calculate the critical exponents for several spin phases that emerge from different initial conditions. In Sec. III, we propose a new master equation that leads to a Metropolis algorithm, which preserves locality and detailed energy balance, also for $q \neq 1$. In Sec. IV, we simulate an equilibrium Ising spin system in a square lattice and show the differences between the results of our approach and those of Refs. [11,12]. Next, we evolve a Ising spin system in a square lattice, from ordered and disordered initial conditions in the context of time-dependent simulations. From such nonequilibrium Monte Carlo simulations, also called short-time simulations, we are able to calculate the dynamic and static critical exponents. Finally, the conclusions are presented in Sec. V.

\section{CRITICAL DYNAMICS OF SPIN SYSTEMS AND TIME-DEPENDENT SIMULATIONS}

Here, we briefly review finite-size scaling in the dynamics relaxation of spin systems. We present our alternative deduction of the some expected power laws in the short-time dynamics context. Readers who want a more complete review about this topic may want to read Ref. [14].

This topic is based on time-dependent simulations, and it constitutes an important issue in the context of phase transitions and critical phenomena. Such methods can be applied not only to estimate the critical parameters in spin systems, but also to calculate the critical exponents (static and dynamic ones) through different scaling relations by setting different initial conditions.

The study of statistical system dynamical critical properties has become simpler in nonequilibrium physics after the seminal ideas of Janssen, Schaub, and Schmittmann [15] and of Huse [16]. Quenching systems from high temperatures to the critical one, they have shown universality and scaling behavior to appear already in the early stages of time evolution, via renormalization-group techniques and numerical calculations, respectively. Hence, using short-time dynamics, one can often circumvent the well known problem of the critical slowing down that plagues investigations of the long-time regime.

The dynamic scaling relation obtained by Janssen et al. for the magnetization $k$ th moment, extended to finite size systems, is written as

$$
\left\langle M^{k}\right\rangle\left(t, \tau, L, m_{0}\right)=b^{-k \beta / v}\left\langle M^{k}\right\rangle\left(b^{-z} t, b^{1 / \nu} \tau, b^{-1} L, b^{x_{0}} m_{0}\right),
$$

where the arguments are the time $t$, the reduced temperature $\tau=\left(T-T_{c}\right) / T_{c}$, with $T_{c}$ being the critical one, the lattice linear size $L$, and initial magnetization $m_{0}$. Here, the operator $\langle\cdots\rangle$ denotes averages over different configurations due to different possible time evolutions from each initial configuration compatible with a given $m_{0}$. On the equation's right-hand side one has an arbitrary spatial rescaling factor $b$ and an anomalous dimension $x_{0}$ related to $m_{0}$. The exponents $\beta$ and $v$ are the equilibrium critical exponents associated with the order parameter and the correlation length, respectively. The exponent $z$ is the dynamic one, which characterizes the time correlations in equilibrium. After the scaling $b^{-1} L=1$ and at the critical temperature $T=T_{c}$, the first $(k=1)$ magnetization moment is $\langle M\rangle\left(t, L, m_{0}\right)=L^{-\beta / v}\langle M\rangle\left(L^{-z} t, L^{x_{0}} m_{0}\right)$.
Denoting $u=t L^{-z}$ and $w=L^{x_{0}} m_{0}$, one has $\langle M\rangle(u, w)=$ $\langle M\rangle\left(L^{-z} t, L^{x_{0}} m_{0}\right)$. The derivative with respect to $L$ is $\partial_{L}\langle M\rangle=(-\beta / v) L^{-\beta / v-1}\langle M\rangle(u, w)+L^{-\beta / v}\left[\partial_{u}\langle M\rangle \partial_{L} u+\right.$ $\left.\partial_{w}\langle M\rangle \partial_{L} w\right]$, where we have explicitly $\partial_{L} u=-z t L^{-z-1}$ and $\partial_{L} w=x_{0} m_{0} L^{x_{0}-1}$. In the limit $L \rightarrow \infty, \partial_{L}\langle M\rangle \rightarrow 0$, one has $x_{0} w \partial_{w}\langle M\rangle-z u \partial_{u}\langle M\rangle-\beta / \nu\langle M\rangle=0$. The separability of the variables $u$ and $w$ in $\langle M\rangle(u, w)=M_{1}(u) M_{2}(w)$ leads to $x_{0} w M_{2}^{\prime} / M_{2}=\beta / v+z u M_{1}^{\prime} / M_{2}$, where the prime means the derivative with respect to the argument. Since this equation's left-hand side depends only on $w$ and the right-hand side depends only on $u$, they must be equal to a constant $c$. Thus, $M_{1}(u)=u^{(c / z)-\beta /(v z)}$ and $M_{2}(w)=w^{c / x_{0}}$, resulting in $\langle M\rangle(u, w)=m_{0}^{c / x_{0}} L^{\beta / v} t^{(c-\beta / v) / z}$. Returning to the original variables, one has $\langle M\rangle\left(t, L, m_{0}\right)=m_{0}^{c / x_{0}} t^{(c-\beta / v) / z}$.

On one hand, choosing $c=x_{0}$ and calculating $\theta=\left(x_{0}-\right.$ $\beta / \nu) / z$, at criticality $(\tau=0)$, we obtain $\langle M\rangle_{m_{0}} \sim m_{0} t^{\theta}$ corresponding to a regime under small initial magnetization. This can be observed by a finite-time scaling $b=t^{1 / z}$ in Eq. (1), at critical temperature $(\tau=0)$ which leads to $\langle M\rangle\left(t, m_{0}\right)=t^{-\beta /(\nu z)}\langle M\rangle\left(1, t^{x_{0} / z} m_{0}\right)$. Defining $x=t^{x_{0} / z} m_{0}$, an expansion of the averaged magnetization around $x=$ 0 results in $\langle M\rangle(1, x)=\langle M\rangle(1,0)+\left.\partial_{x}\langle M\rangle\right|_{x=0} x+O\left(x^{2}\right)$. By construction $\langle M\rangle(1,0)=0$, since $u=t^{x_{0} / z} m_{0} \ll 1$ and $\left.\partial_{x}\langle M\rangle\right|_{x=0}$ is a constant. So, by discarding the quadratic terms we obtain the expected power-law behavior $\langle M\rangle_{m_{0}} \sim m_{0} t^{\theta}$. This anomalous behavior of initial magnetization is valid only for a characteristic time scale $t_{\max } \sim m_{0}^{-z / x_{0}}$.

On the other hand, the choice $c=0$ corresponds to a case where the system does not depend on the initial trace of the system, and $m_{0}=1$ leads to the simple power law

$$
\langle M\rangle_{m_{0}=1} \sim t^{-\beta /(v z)}
$$

that similarly corresponds to decay of magnetization for $t>$ $t_{\text {max }}$ of a system that previously evolved from a initial small magnetization $\left(m_{0}\right)$, and had its magnetization increased up to a magnetization peak.

For $m_{0}=0$, it is not difficult to show that the magnetization second moment is

$$
\left\langle M^{2}\right\rangle_{m_{0}=0} \sim t^{(d-2 \beta / v) / z}
$$

where $d$ is the system dimension.

Using Monte Carlo simulations, many authors have obtained the dynamic exponents $\theta$ and $z$ as well as the static ones $\beta$ and $\nu$, and other specific exponents for many different models and situations: Baxter-Wu [17], two-, three-, and four-state Potts [18,19], Ising with multispin interactions [20], models with no defined Hamiltonian (celular automata and contact process) [21-23], models with tricritical point [24], Heisenberg [25], protein folding [26,27], and propagation of damages in Ising models [28].

The sequence to determine the static exponents from shorttime dynamics is to determine $z$ first, performing Monte Carlo simulations that mix initial conditions [18], and consider the power law for the cumulant

$$
F_{2}(t)=\frac{\left\langle M^{2}\right\rangle_{m_{0}=0}}{\langle M\rangle_{m_{0}=1}^{2}} \sim t^{d / z} .
$$


Once $z$ is calculated, the exponent $\eta=2 \beta / v$ is calculated according to $\eta=2(\widehat{\beta / \nu} z) \cdot \widehat{z}$, where $(\widehat{\beta / \nu} z)$ was estimated via magnetization decay and $\widehat{z}$ from cumulant $F_{2}$.

However, prior to obtaining the critical exponents, we also perform time-dependent MC simulations in order to refine the critical temperatures. These are based on power laws obtained by finite-size scaling analysis of the magnetization decay from an initially ordered state Eq. (2). This choice demands a number of runs smaller than other power laws in nonequilibrium, and so we propose a simple algorithm that spans different critical values to find the best determination coefficient in the linear fit $\ln \langle M\rangle$ versus $\ln t$. This procedure is explored in Sec. IV, and is used later to calculate the critical temperatures for Ising models with different values of the nonextensivity parameter $q$ in our new Metropolis prescription.

\section{GENERALIZED MASTER EQUATION}

In this section, we start recalling the way that the Metropolis algorithm is obtained from the master equation for spin systems. We point out that the energy difference caused by flipping an Ising spin is local, i.e., it depends only on the flipped spin. Next, we show a first attempt to generalize the Metropolis algorithm [11,12], according to the nonextensive thermostatistics, introduced by Tsallis [1]. We show that this generalization does not preserve the spin flip locality. To recover this locality, we propose a new generalized master equation, which leads to a different generalization of the Metropolis algorithm.

\section{A. Standard master equation and Metropolis algorithm}

In general, spin system nonequilibrium dynamics is described by the time evolution of the probability $P(E, t)$ that, at instant $t$, the system has an energy $E$. This probability is obtained from the master equation $d P\left(E^{(a)}, t\right) / d t=\sum_{\sigma_{i}^{(b)}}\left\{w\left[\sigma_{i}^{(b)} \rightarrow \sigma_{i}^{(a)}\right] P\left[E^{(b)}, t\right]-\right.$ $\left.w\left[\sigma_{i}^{(a)} \rightarrow \sigma_{i}^{(b)}\right] P\left[E^{(a)}, t\right]\right\}$, where $w\left[\sigma_{i}^{(b)} \rightarrow \sigma_{i}^{(a)}\right]$ is the transition rate of the $i$ th spin from $\sigma_{i}^{(b)}$ to $\sigma_{i}^{(a)}$. Here, $E^{(b)}\left(E^{(a)}\right)$ is the energy of the system before (after) the transition. As $t \rightarrow \infty, d P(E, t) / d t=0$ is a necessary condition for equilibrium. A sufficient but not necessary condition for equilibrium, known as detailed balance condition, supposes a more restricted situation for occurrence of $d P(E, t) / d t=$ 0 , i.e., $w\left[\sigma_{i}^{(b)} \rightarrow \sigma_{i}^{(a)}\right] P\left[E^{(b)}\right]-w\left[\sigma_{i}^{(a)} \rightarrow \sigma_{i}^{(b)}\right] P\left[E^{(a)}\right]=0$, meaning that each term in the summation vanishes. In this case, $P(E)=P(E, t \rightarrow \infty)$ is the Boltzmann distribution: $P\left(E_{j}\right)=e^{-\beta E_{j}} / \sum_{k} e^{-\beta E_{k}}$, where the summation is over the different energy states and $\beta=\left(k_{B} T\right)^{-1}$.

Employing detailed balance requires one to find simple prescriptions for spins system dynamics, as for example the Metropolis prescription: $w\left[\sigma_{i}^{(b)} \rightarrow \sigma_{i}^{(a)}\right]=$ $\min \left\{1, \exp \left[-\beta\left(E^{(a)}-E^{(b)}\right)\right]\right\}$. When applied to evolve spin systems, this simple dynamics reduces to calculating just local energy changes. For instance, the Ising model in two dimensions has an energy $E^{(b)}=-J \sigma_{i_{x}, i_{y}}^{(b)} S_{i_{x}, i_{y}}+\xi$ before the flip of spin $\sigma_{i_{x}, i_{y}}$, located at the site indexed by $i_{x}$ and $i_{y}$, where the local energy change is quantified by

$$
S_{i_{x}, i_{y}}=\sigma_{i_{x}+1, i_{y}}+\sigma_{i_{x}-1, i_{y}}+\sigma_{i_{x}, i_{y}-1}+\sigma_{i_{x}, i_{y}+1}
$$

and the nonlocal energy is $\xi$, which is obtained excluding the spin $\sigma_{i_{x}}, i_{y}$ from the calculation. After the spin flip, the energy is $E^{(a)}=-J \sigma_{i_{x}, i_{y}}^{(a)} S_{i_{x}, i_{y}}+\xi$ and the energy change of the system due to the spin $\sigma_{i_{x}, i_{y}}$ flip is simply

$$
E^{(a)}-E^{(b)}=-J\left[\sigma_{i_{x}, i_{y}}^{(a)}-\sigma_{i_{x}, i_{y}}^{(b)}\right] S_{i_{x}, i_{y}},
$$

which does not depend on the energy of the other spins.

\section{B. Generalized Metropolis algorithm}

The system equilibrium is described by the generalized Boltzmann-Gibbs distribution

$$
P_{1-q}\left(E_{i}\right)=\frac{\left[e_{1-q}\left(-\beta^{\prime} E_{i}\right)\right]^{q}}{\sum_{i=1}^{\Omega}\left[e_{1-q}\left(-\beta^{\prime} E_{i}\right)\right]^{q}},
$$

where $\Omega$ is the number of accessible states of the system and $\beta^{\prime}=\beta / \sum_{i=1}^{\Omega}\left\{\left[e_{1-q}\left(-\beta E_{i}\right)\right]^{q}+(1-q) \beta\langle E\rangle_{1-q}\right\}$, where $\langle E\rangle_{1-q}=\sum_{i=1}^{\Omega} E_{i} P_{1-q}\left(E_{i}\right)$. Here it is important to mention that $\left(k_{B} \beta^{\prime}\right)^{-1}$ is a scale temperature that can be used to interpret experimental and computational experiments. There is a heated ongoing discussion whether it is the physical temperature or not.

The function

$$
e_{\alpha}(x)= \begin{cases}(1+\alpha x)^{1 / \alpha} & \text { for } \alpha x>-1, \\ 0 & \text { otherwise, }\end{cases}
$$

is the generalized exponential [29,30]. For $\alpha \rightarrow 0$, one retrieves the standard exponential function $e_{0}(x)=e^{x}$. It is this singularity at $\alpha x>-1$ that brings up interesting effects such the survival and extinction transitions in onespecies population dynamical models [8]. The inverse of the generalized exponential function is the generalized logarithmic function $\ln _{\alpha}(x)=\left(x^{\alpha}-1\right) / \alpha$, which for $\alpha \rightarrow 0$ leads to the standard logarithm function $\ln _{0}(x)=\ln (x)$. Notice that the inequality $\alpha x>-1$ for fixed $x$ produces a limiting value for $\alpha$. This generalized logarithmic function has been introduced first in the context of nonextensive thermostatistics $[1,29]$ and has a clear geometrical interpretation as the area between 1 and $x$ underneath the nonsymmetric hyperbola $1 / t^{1-\alpha}$ [30]. It is interesting to notice that in 1984 Cressie and Read [31] proposed an entropy that would lead to a generalization of the logarithm function given by $\ln _{\alpha}(x) /(\alpha+1)$. In this case, we would gain the limiting value in $\alpha$ but lose its geometrical interpretation.

To recover the additive property of the argument, when multiplying two generalized exponential functions $e_{\alpha}(a) e_{\alpha}(b)=$ $e_{\alpha}\left(a \oplus_{\alpha} b\right)\left[e_{\alpha}(a) / e_{\alpha}(b)=e_{\alpha}\left(a \ominus_{\alpha} b\right)\right]$ and $e_{\alpha}(a) \otimes_{\alpha} e_{\alpha}(b)=$ $e_{\alpha}(a+b)\left[e_{\alpha}(a) \oslash_{\alpha} e_{\alpha}(b)=e_{\alpha}(a-b)\right]$ consider the following algebraic operators $[32,33]$ :

$$
\begin{aligned}
& a \oplus_{\alpha} b=a+b+\alpha a b, \\
& a \ominus_{\alpha} b=\frac{a-b}{1+\alpha b}, \\
& a \otimes_{\alpha} b=\left(a^{\alpha}+b^{\alpha}-1\right)^{1 / \alpha}, \\
& a \oslash_{\alpha} b=\left(a^{\alpha}-b^{\alpha}+1\right)^{1 / \alpha} .
\end{aligned}
$$



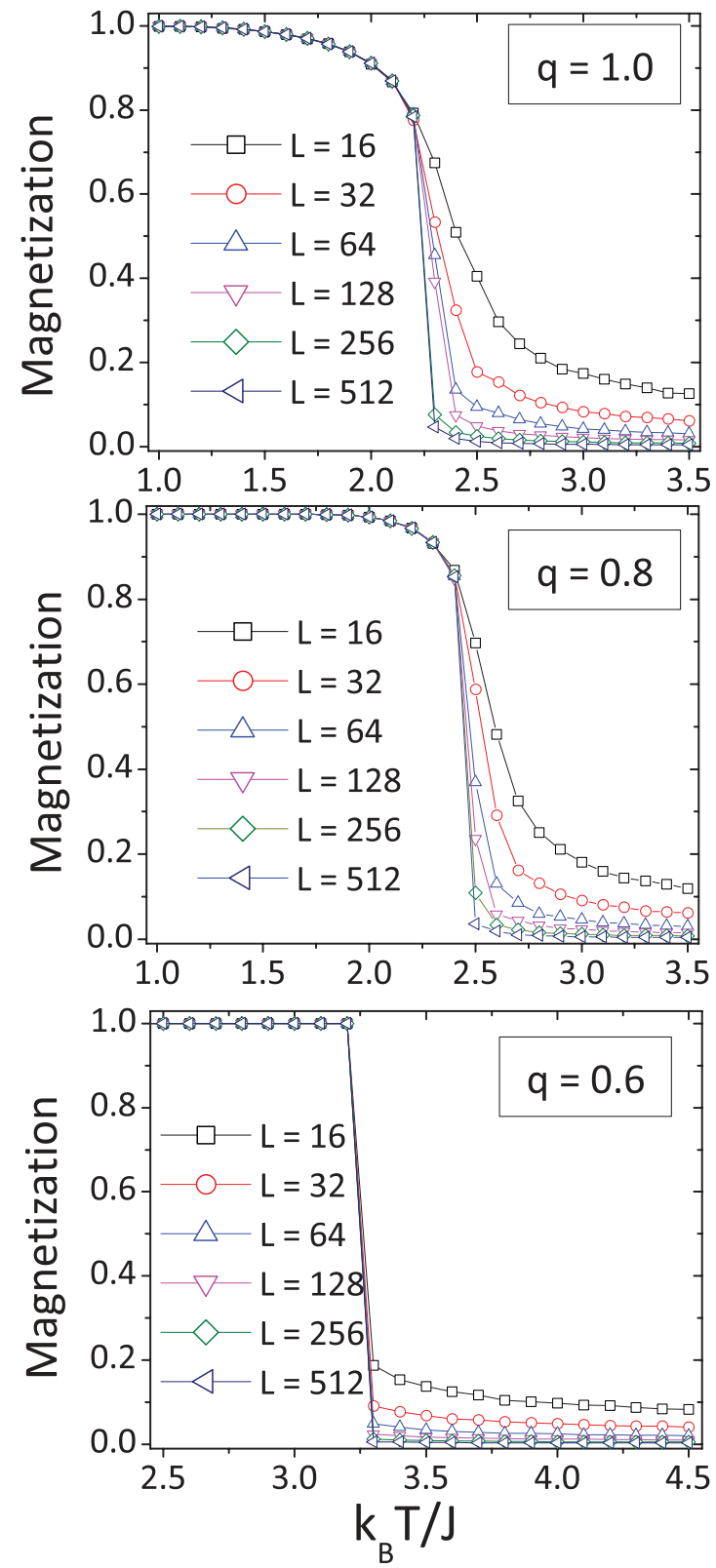

FIG. 1. (Color online) System magnetization versus temperature for $q=1.0,0.8$, and 0.6. Using the dynamics based on Metropolis II, we observe phase transitions for critical values up to $\ln (1+\sqrt{2}) / 2$ as $q<1$, different from previous studies, which are based on Metropolis I.

Observe that if $a \ominus_{\alpha} b=0$ then $a=b$, and if $a \otimes_{\alpha} b=c \otimes_{\alpha} d$ then $a \oslash_{\alpha} c=d \oslash_{\alpha} b$.

However, in equilibrium, the Ising model prescribes an adapted Metropolis dynamics that considers a generalized version of the exponential function $[11,12]$

$$
w\left[\sigma_{i}^{(b)} \rightarrow \sigma_{i}^{(a)}\right]=\frac{P_{1-q}\left[E^{(a)}\right]}{P_{1-q}\left[E^{(b)}\right]}=\left\{\frac{e_{1-q}\left[-\beta^{\prime} E^{(a)}\right]}{e_{1-q}\left[-\beta^{\prime} E^{(b)}\right]}\right\}^{q} .
$$

From the generalization of the exponential function in the Boltzmann-Gibbs weight, the transition rate of Eq. (12) can be used to determine the system evolution as the Metropolis algorithm. Nevertheless, we stress that, in such a choice, the dynamics is not local. Because generalized exponential
TABLE I. Comparison between critical the critical temperature and error, for the two-dimensional (2D) Ising model, obtained from extrapolation $L \rightarrow \infty$ (see Fig. 2) using the algorithm of Ref. [11] (Metropolis I) and our algorithm (Metropolis II).

\begin{tabular}{lcc}
\hline \hline$q$ & Metropolis I & Metropolis II \\
\hline 0.6 & $1.761(3)$ & $3.201(1)$ \\
0.8 & $1.891(7)$ & $2.461(5)$ \\
1.0 & $2.259(11)$ & $2.262(9)$ \\
\hline \hline
\end{tabular}

functions are nonadditive, a spin flip introduces a change in the system energy that is spread all over the lattice. More precisely, consider the Ising model in a square lattice. One can show that

$$
\frac{e_{1-q}\left[-\beta^{\prime} E^{(a)}\right]}{e_{1-q}\left[-\beta^{\prime} E^{(b)}\right]}=e_{1-q}\left\{-\beta^{\prime}\left[E^{(a)} \ominus_{1-q} E^{(b)}\right]\right\}
$$

or

$$
\frac{e_{1-q}\left[-\beta^{\prime} E^{(a)}\right]}{e_{1-q}\left[-\beta^{\prime} E^{(b)}\right]} \neq e_{1-q}\left\{-\beta^{\prime}\left[E^{(a)}-E^{(b)}\right]\right\}
$$

where $E^{(a)}-E^{(b)}$ is given by Eq. (5), which depends only the spins that directly interact with the flipped spin, violating the detailed energy balance.

In Refs. [11,12], the authors consider (with no explanations) the equality in Eq. (14), instead of considering Eq. (13). Thus, the detailed energy balance is violated, since the system is updated following a local calculation of the generalized Metropolis algorithm of Eq. (12).

To correct this problem, one must update the spin system using the nonlocality of Eq. (12), which is numerically expensive, since the energy of the whole lattice must be recalculated due to a simple spin flip. The other alternative is to require that the transition rate depend locally on the energy difference of a simple spin flip, which in turn leads us to a modified master equation. Since the former is very expensive numerically, we explore only the latter alternative which is numerically faster and is able to produce statistically significant results for fairly large spin systems.

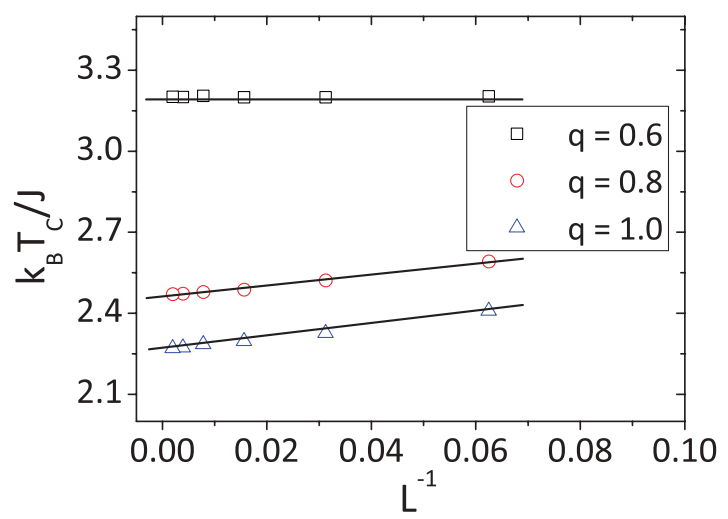

FIG. 2. (Color online) Extrapolation $(L \rightarrow \infty)$ of critical temperatures for different $q$ values: $0.6,0.8$, and 1.0 for the 2D Ising model. 
TABLE II. Coarse-grained stage for Metropolis I. The values of determination coefficient $\alpha$ of the linear fit $\ln \langle M\rangle$ versus $\ln t$ for different $q$ values. The highest values are in bold and correspond to best critical temperature found at the first stage (coarse grained). For example, for $q=0.75$, the best $r$ is 0.940558872 , which corresponds to $k_{B} T_{c}^{(1)} / J=1.86918531$.

\begin{tabular}{|c|c|c|c|c|c|c|c|}
\hline$k_{B} T_{c}^{(1)} / J$ & $q=0.70$ & $q=0.75$ & $q=0.80$ & $q=0.85$ & $q=0.90$ & $q=0.95$ & $q=1.00$ \\
\hline$T^{*}-0.6$ & 0.644974839 & 0.544533372 & 0.433158672 & 0.376097284 & 0.326410245 & 0.282451042 & 0.263459517 \\
\hline$T^{*}-0.5$ & 0.998967222 & 0.872350719 & 0.65553425 & 0.492266999 & 0.392016875 & 0.336915743 & 0.306414971 \\
\hline$T^{*}-0.4$ & 0.858060019 & 0.940558872 & 0.979041762 & 0.731374836 & 0.500535816 & 0.396550709 & 0.341806493 \\
\hline$T^{*}-0.3$ & 0.822853648 & 0.82063843 & 0.90326648 & 0.999101612 & 0.773207193 & 0.535343676 & 0.409044416 \\
\hline$T^{*}-0.2$ & - & - & 0.833548876 & 0.885834994 & 0.998950355 & 0.788883669 & 0.547803953 \\
\hline$T^{*}-0.1$ & - & - & - & 0.836458324 & 0.882565862 & 0.999817616 & 0.776353951 \\
\hline$T^{*}=\ln (1+\sqrt{2}) / 2$ & - & - & - & - & 0.817075651 & 0.897612219 & 0.997114577 \\
\hline$T^{*}+0.1$ & - & - & - & - & - & 0.82435859 & 0.916225167 \\
\hline
\end{tabular}

\section{Recovering locality in the generalized Metropolis algorithm}

Based on the operators of Eqs. (8)-(11), we propose the following generalized master equation:

$$
\begin{aligned}
\frac{d P_{1-q}\left[E^{(a)}\right]}{d t}= & \sum_{\sigma_{i}^{(b)}} w\left[\sigma_{i}^{(b)} \rightarrow \sigma_{i}^{(a)}\right] \otimes_{\tilde{q} / q} P_{q}\left[E^{(b)}\right] \ominus_{\tilde{q} / q} \\
& \times w\left[\sigma_{i}^{(a)} \rightarrow \sigma_{i}^{(b)}\right] \otimes_{\tilde{q} / q} P_{q}\left[E^{(a)}\right] .
\end{aligned}
$$

where $P_{q}(E)$ is given by Eq. (6). Here, it is suitable to call $\tilde{q}=$ $1-q$ and write the generalized exponentials as a function of $\tilde{q}$. In equilibrium, $d P_{1-q} / d t=0$ and the dynamics is governed by Eq. (6).

The detailed balance (a sufficient condition for equilibrium) for the generalized master equation is

$$
\begin{aligned}
& w\left[\sigma_{i}^{(b)} \rightarrow \sigma_{i}^{(a)}\right] \oslash_{\tilde{q} / q} w\left[\sigma_{i}^{(a)} \rightarrow \sigma_{i}^{(b)}\right] \\
& =P_{q}\left[E^{(a)}\right] \oslash_{\tilde{q} / q} P_{q}\left[E^{(b)}\right],
\end{aligned}
$$

which leads to a new generalized Metropolis algorithm

$$
\begin{aligned}
w\left(\sigma_{i}^{(b)} \rightarrow \sigma_{i}^{(a)}\right) & =\min \left\{1,\left[e_{\tilde{q}}\left(-\beta^{\prime} E^{(a)}\right)\right]^{q} \oslash_{\tilde{q} / q}\left[e_{\tilde{q}}\left(-\beta^{\prime} E^{(b)}\right)\right]^{q}\right\} \\
& =\min \left\{1,\left[e_{\tilde{q}}\left(-\beta^{\prime}\left(E^{(a)}-E^{(b)}\right)\right)\right]^{q}\right\} \\
& =\min \left\{1,\left[e_{\tilde{q}}\left(\beta^{\prime} J\left[\sigma_{i_{x}, i_{y}}^{(a)}-\sigma_{i_{x}, i_{y}}^{(b)}\right] S_{i_{x}, i_{y}}\right)\right]^{q}\right\},
\end{aligned}
$$

and now the transition probability depends only on the energy between the read site and its neighbors; i.e., locality is retrieved.

\section{GENERALIZED METROPOLIS ALGORITHM: NUMERICAL SIMULATION RESULTS}

We have performed Monte Carlo simulations of the square lattice Ising model in the context of generalized BoltzmannGibbs weights. These simulations are based on two approaches for Metropolis dynamics. The first one (Metropolis I) is described in Ref. [11], where the nonlocal transition rate of Eq. (12) is used to update the spin system. In the second approach (Metropolis II), the local transition rate of Eq. (17) is used. We separate our results into two different subsections, for the equilibrium simulations and short-time critical dynamics.

\section{A. Equilibrium}

In this part we analyze the magnetization $\langle m\rangle$, where $\langle\cdot\rangle$ denotes averages under Monte Carlo (MC) steps. We perfom MC simulations for $q=0.6, q=0.8$, and $q=1.0$. In the simulations, we have used $L_{\min }=2^{4}=16$ up to $L_{\max }=2^{9}=$ 512 , with periodic boundary conditions and a random initial configuration of the spins with $\left\langle m_{0}\right\rangle=0$. Differently from what was reported in Ref. [11], where the results have been obtained after $10^{7} \mathrm{MC}$ steps per spin, we have used $6.1^{3} \mathrm{MC}$ steps per spin, an equilibrium situation consistent with the one reported by Newman and Barkema [34]. This results in $1.5 \times 10^{6}-1.5 \times 10^{9} \mathrm{MC}$ steps for the whole lattice of $16^{2} \mathrm{up}$ to $512^{2}$ spins.

Figure 1 shows the magnetization curves as functions of critical temperature for different $q$ values. The critical temperature increases as $q$ decreases. This behavior, using our algorithm (Metropolis II) differs from the one obtained using the algorithm of Refs. [11,12] (Metropolis I). We stress that

TABLE III. Critical temperature and exponents obtained for different $q$ values for prescription Metropolis I. The exponents where obtained performing simulations for the estimated critical temperatures and were based on power laws previously described in the short-time regime. The last line shows the $r$ value for the best fits in the second stage (fine scale).

\begin{tabular}{llllllll}
\hline \hline$q$ & \multicolumn{1}{c}{0.70} & \multicolumn{1}{c}{0.75} & \multicolumn{1}{c}{0.80} & \multicolumn{1}{c}{0.85} & 0.90 & 0.95 & \multicolumn{1}{c}{1.00} \\
\hline$k_{B} T_{c}^{(2)} / J$ & $1.77(1)$ & $1.82(1)$ & $1.89(1)$ & $1.97(1)$ & $2.07(1)$ & $2.17(1)$ & $2.27(1)$ \\
$\beta / \nu z$ & $0.060(4)$ & $0.062(7)$ & $0.078(5)$ & $0.082(2)$ & $0.100(5)$ & $0.094(4)$ & $0.057(3)$ \\
$z$ & $2.13(4)$ & $2.15(5)$ & $2.12(4)$ & $2.09(3)$ & $2.10(3)$ & $2.11(6)$ & $2.15(3)$ \\
$\theta$ & $0.18(4)$ & $0.14(4)$ & $0.22(7)$ & $0.17(3)$ & $0.04(6)$ & $0.17(3)$ & $0.19(4)$ \\
$\eta$ & $0.25(2)$ & $0.27(3)$ & $0.33(2)$ & $0.34(1)$ & $0.42(2)$ & $0.40(2)$ & $0.25(1)$ \\
$r$ & 0.998568758 & 0.998915473 & 0.999342437 & 0.999458152 & 0.999589675 & 0.999718708 & 0.999206853 \\
\hline
\end{tabular}



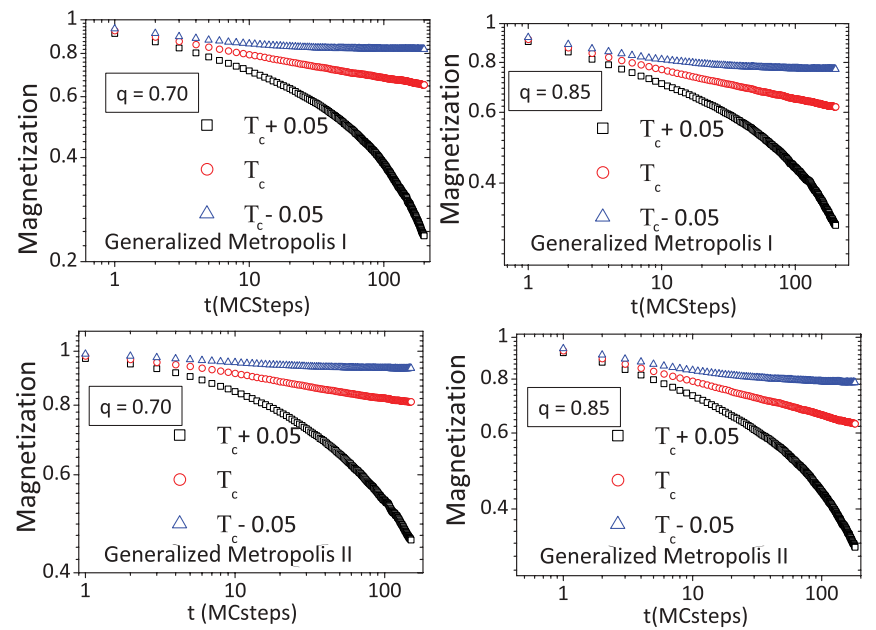

FIG. 3. (Color online) Decay of magnetization according to the power law $M(t) \sim t^{-\beta / v z}$ at the critical temperature found by the considered algorithms (red circles), for $q=0.70$ and 0.85 . We also show the plots considering MC simulations for $T_{c}+\delta$ and $T_{c}-\delta$. We used $\delta=0.05$. The upper (lower) plots correspond to the Metropolis I (II) algorithm.

both algorithms agree for $q=1$, the usual Boltzmann-Gibbs weights, converging to the theoretical value $\ln (1+\sqrt{2}) / 2$. In Table I, we show the critical temperature and error obtained from the extrapolation $L \rightarrow \infty$ (see Fig. 2) using both algorithms. These results suggest a thorough difference among the processes and critical values found between two the dynamics Metropolis I and II. In Fig. 1, the curves show phase transitions for critical values up to $\ln (1+\sqrt{2}) / 2$ as $q<1$. This differs from previous studies, which are based on prescription Metropolis I.

Fig. 1 shows that, differently from the $q=0.8$ and $q=$ 1.0 cases, for $q=0.6$ the discontinuity in the magnetization curve does not depend on system size $L$. In fact, in this case, the critical temperature $T_{c}$ does not depend on $L$. This effect occurs due to the cutoff of the escort probability distribution as reported for Metropolis I [11] for $q<0.5$. For Metropolis II, Fig. 2 depicts that $T_{c}$ remains constant for all values of $L^{-1}$, for $q=0.6$. For both cases, $q=1.0$ (obviously) and $q=0.8$, we have verified that $v \approx 1$ and $\beta \approx 0.125$, obtained from the collapse of the curves $\langle M\rangle L^{\beta / v}$ versus $\left(T-T_{c}\right) L^{1 / v}$. This data collapse permits the extrapolation of $k_{B} T_{c} / J$ versus $L^{-1}$, since $v \approx 1$ for both cases according to Fig. 2 . In the following, we show using nonequilibrium simulations that $2 \beta / v \approx 0.25$, for $q=1$ and $q=0.8$, validating the data collapse results (see Table V).

Another important question to be formulate is: Can we corroborate the same behavior in nonequilibrium simulations? In the next section, we show results from MC simulations in the nonequilibrium regime under the two dynamics (Metropolis I and II). We also analyze the critical exponents (dynamic and static) as a function $q$ from short-time dynamics. We show that short-time dynamics corroborate the behavior predicted by two dynamics, suggesting that Metropolis II indeed presents an increase of critical value as $q$ value increases, different from Metropolis I. Our results suggest that these techniques based on time-dependent simulations can be extended also for $q \neq 1$, in short-range spin models.

\section{B. Short time}

Here we address time-dependent MC simulations in the context of so-called short-time dynamics. First, to test our methodology, we show that critical values obtained from nonequilibrium simulations using Metropolis I must corroborate the critical values obtained in Ref. [11], where MC simulations at equilibrium have been employed. We have checked it. Nevertheless, as in the equilibrium numerical simulations, we show that Metropolis II leads to different values than the Metropolis I method.

Our algorithm to estimate the critical temperature is divided in two stages. In the first stage, a coarse-grained calculation is performed to estimate the critical temperature $T_{c}(q)$, for different $q$ values. In the second stage, one uses the estimated critical temperature obtained in the first stage to run a nonequilibrium Monte Carlo simulation. We denote the second state as fine scale stage. In this stage, one determines the dynamical critical exponent from the short-time behavior of the spin system, as described in Sec. II. Since, even using nonextensive thermostatistics, the magnetization must behave as a power law $\langle M\rangle \sim t^{-\beta / v z}$, we conjecture that changing $T_{c}(q)$ from $T_{c}^{(\min )}(q)$ up to $T_{c}^{(\max )}(q)$, the best $T_{c}(q)$ is the one that leads to the best linear behavior of $\ln \langle M\rangle$ versus $\ln t$. We have considered $n_{s}=500$ realizations, with initial magnetization $m_{0}=1$.

From the theoretical critical temperature $\left[\beta_{c}=J / k_{B} T_{c}=\right.$ $\ln (1+\sqrt{2}) / 2]$, one allows the temperature to vary in the range from $k_{B} T_{c} / J-1$ up to $k_{B} T_{c} / J+1$, setting $k_{B} T / J=$ $[2-\ln (1+\sqrt{2})][\ln (1+\sqrt{2})]+j \Delta$, where $\Delta=0.1$ and

TABLE IV. Coarse-grained stage for Metropolis II. The values of determination coeficient $r$ of the linear fit $\ln \langle M\rangle$ versus $\ln t$, for different $q$ values. As in Table II, the highest values in bold correspond to the best critical temperature found at the first stage (coarse grained).

\begin{tabular}{|c|c|c|c|c|c|c|c|}
\hline$k_{B} T_{c}^{(1)} / J$ & $q=0.70$ & $q=0.75$ & $q=0.80$ & $q=0.85$ & $q=0.90$ & $q=0.95$ & $q=1.00$ \\
\hline$T^{*}-0.1$ & - & 0.369354816 & 0.393077206 & 0.473151224 & 0.555233203 & 0.664145691 & 0.754299904 \\
\hline$T^{*}=\ln (1+\sqrt{2}) / 2$ & - & 0.439789081 & 0.50595381 & 0.658725599 & 0.829581264 & 0.952694449 & 0.997206853 \\
\hline$T^{*}+0.1$ & - & 0.579484235 & 0.756653731 & 0.959530136 & 0.995694839 & 0.951627799 & 0.91284158 \\
\hline$T^{*}+0.2$ & 0.601895662 & 0.836896384 & 0.999315534 & 0.932708995 & 0.869271327 & 0.848547425 & 0.838519074 \\
\hline$T^{*}+0.3$ & 0.844382176 & 0.989767198 & 0.875131078 & 0.833730495 & 0.831169795 & 0.799709762 & - \\
\hline$T^{*}+0.4$ & 0.989716381 & 0.847004746 & 0.812106454 & - & - & - & - \\
\hline$T^{*}+0.5$ & 0.828738110 & 0.787203767 & - & - & - & - & - \\
\hline$T^{*}+0.6$ & 0.842827863 & - & - & - & - & - & - \\
\hline
\end{tabular}


TABLE V. Critical temperature and exponents obtained for different $q$ values for the Metropolis II algorithm. The exponents were obtained performing simulations for the estimated critical temperatures. They were based on power laws described in Sec. II. The last line shows the $r$ value for the best fits in the second stage (fine scale).

\begin{tabular}{llllllll}
\hline \hline$q$ & \multicolumn{1}{c}{0.70} & \multicolumn{1}{c}{0.75} & \multicolumn{1}{c}{0.80} & \multicolumn{1}{c}{0.85} & 0.90 & 0.95 & 1.00 \\
\hline$k_{B} T_{c}^{(2)} / J$ & $2.66(1)$ & $2.55(1)$ & $2.47(1)$ & $2.41(1)$ & $2.36(1)$ & $2.31(1)$ & $2.27(1)$ \\
$\beta / \nu z$ & $0.019(5)$ & $0.039(5)$ & $0.060(4)$ & $0.094(6)$ & $0.116(7)$ & $0.075(4)$ & $0.057(3)$ \\
$z$ & $1.97(4)$ & $2.02(3)$ & $2.10(3)$ & $2.09(3)$ & $2.09(6)$ & $2.20(4)$ & $2.15(3)$ \\
$\theta$ & $0.43(3)$ & $0.21(7)$ & $0.22(3)$ & $0.11(4)$ & $0.16(5)$ & $0.13(3)$ & $0.19(4)$ \\
$\eta$ & $0.07(2)$ & $0.16(2)$ & $0.25(2)$ & $0.39(3)$ & $0.48(3)$ & $0.33(2)$ & $0.25(1)$ \\
$r$ & 0.994455464 & 0.998375667 & 0.999227272 & 0.999226704 & 0.99928958 & 0.99925661 & 0.997206853 \\
\hline \hline
\end{tabular}

$j=0,1, \ldots, 20$. This is the coarse-grained stage. For each temperature, a linear fit is performed and one calculates the determination coefficient of fit as

$$
r=\frac{\sum_{t=1}^{N_{M C}}(\overline{\ln \langle M\rangle}-a-b \ln t)^{2}}{\sum_{t=1}^{N_{M C}}(\overline{\ln \langle M\rangle}-\ln \langle M\rangle(t))^{2}},
$$

and $\overline{\ln \langle M\rangle}=\left(1 / N_{M C}\right) \sum_{t=1}^{N_{M C}} \ln \langle M\rangle(t)$, where $N_{M C}$ is the number of Monte Carlo sweeps. In our experiments, we have used $N_{M C}=300 \mathrm{MC}$ steps. Here, $r=1$ means an exact fit, so that the closer $r$ is to unity, the better. Here, $a$ and $b$ are the linear coefficient and the slope in the linear fit $\ln \langle M\rangle$ versus $\ln t$, respectively. From $b$, one estimates the exponent $-\beta v / z$.

In the fine-scale stage, we refine the critical temperature $k_{B} T_{c}^{(1)}(q) / J$ obtained in the first stage. We use the algorithm considering $\Delta=0.01$, with $j=0,1, \ldots, 20$ considering $k_{B} T_{c}^{(2)}(q) / J=k_{B} T_{c}^{(1)}(q) / J-0.1+j \Delta$ now to find the best critical temperature in the range from $k_{B} T_{c}^{(1)}(q) / J-0.1$ to $k_{B} T_{c}^{(1)}(q) / J+0.1$ with precision $\Delta=0.01$.

A natural validation for our algorithm is to reproduce the results obtained in Ref. [11], in equilibrium, using the Metropolis I approach, for a specific $q$ value, considering our MC nonequilibrium simulations. For instance, for $q=$ 0.70 , one has at equilibrium $k_{B} T_{c} / J=1.891(7)$ in Ref. [11]. After two stages, our algorithm produces $k_{B} T_{c}^{(2)} / J=1.889$, validating our numerical code.

Next, we use the algorithm with the following values: $q=0.70,0.75,0.80,0.85,0.90,0.95$, and 1.00 , in the equilibrium situation. In Table II, we show our results for the first stage (coarse grained) using the Metropolis I prescription. The values of the determination coefficient $\alpha$ of the linear fit $\ln \langle M\rangle$ versus $\ln t$ are presented for different $q$ values. The highest values (in bold) correspond to best critical temperature found in the first stage. For example, for $q=0.75$, we find that the best $\alpha$ value is 0.940558872 , which corresponds to $k_{B} T_{c}^{(1)}(q) / J=1.86918531$.

In Table II, the symbol "-_" corresponds to situations where the computation of slopes is not possible, due to large deviations in magnetization.

After the refinement (second stage), the best values found for the critical temperatures using the Metropolis I prescription for different $q$ values are presented in the first line of Table III. In Fig. 3, for $q=0.70$ and 0.85 , we show the magnetization decays as the power law $M(t) \sim t^{-\beta / v z}$, for the critical temperature estimated using our algorithm (Metropolis II) and the Metropolis I algorithm. Also, we show the plots considering $\mathrm{MC}$ simulations for $T_{c}+\delta$ and $T_{c}-\delta$, with $\delta=0.05$.

We use the same procedure to find the critical temperatures for prescription Metropolis II. We find very different results, when compared with the ones obtained with Metropolis I. Similarly to Table II, we show the results using the Metropolis II prescription in Table IV. The values are smaller than the ones found with the Metropolis I prescription. However, they match as $q \rightarrow 1$, which validates the numerical procedure.

Similarly, the best results after the fine-scale refinement (second stage) are shown in the first line of Table V.

The magnetization decay obtained by the Metropolis II algorithm is depicted in Fig. 3. After obtaining these estimates for the critical temperatures, we perform short-time simulations to obtain the critical dynamic exponents $z$ and $\theta$ and the static one $\eta=2 \beta / \nu$, using the power laws of Sec. II. Here, we calculated $\theta$ from time correlation $C(t)=\langle M(t) M(0)\rangle$. Tomé and de Oliveira [35] showed that correlation behaves as $C(t) \sim t^{\theta}$, where $\theta$ is exactly the same exponent from initial slope of magnetization from lattices prepared with initial fixed magnetization $m_{0}$. The advantage of this method is that we repeat $N_{s}$ runs, but the lattice does not require a fixed initial magnetization. It is enough to choose the spin with probability $1 / 2$, i.e., $m_{0}=0$ in average. This method does not require the extrapolation $m_{0} \rightarrow 0$.
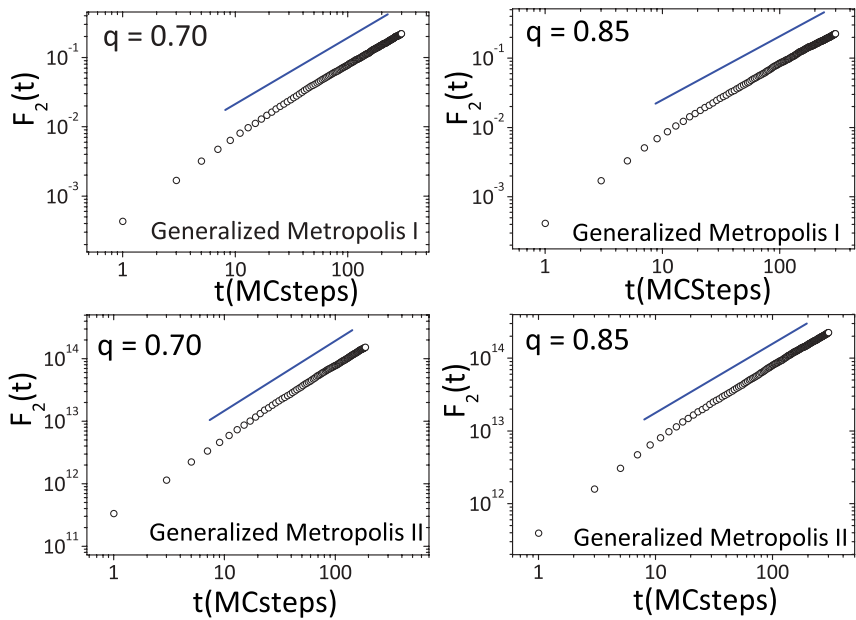

FIG. 4. (Color online) Dynamic cumulant $F_{2}(t)$ versus $t$ in logarithmic scale. The slope gives $d / z$ which supplies the $z$ value. Both prescriptions (Metropolis I and II) are studied. 

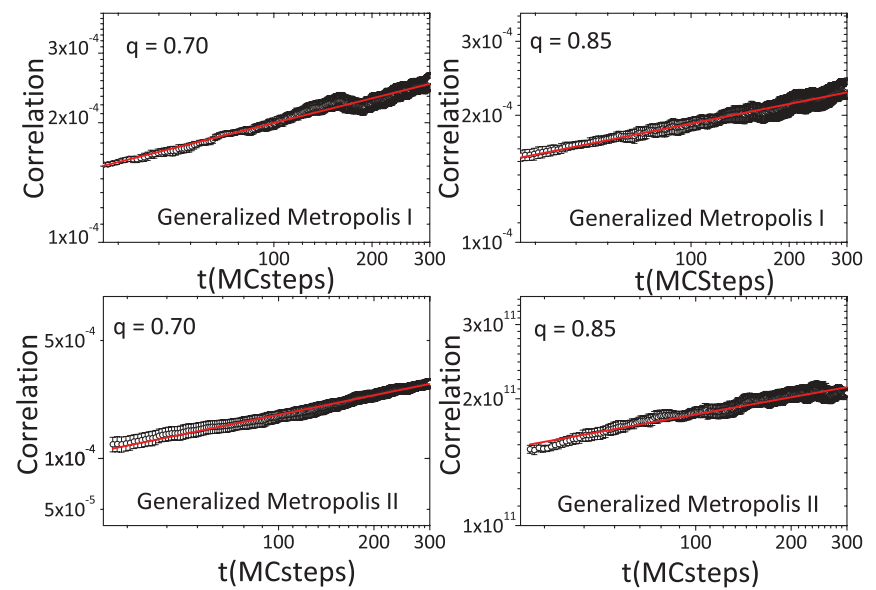

FIG. 5. (Color online) Time correlation of magnetization $C(t)=\langle M(t) M(0)\rangle$ for two prescriptions: Metropolis I and II.

Figures 4 and 5 depict plots of time evolution of $F_{2}$ of Eq. (4) and $C(t)$ as functions of $t$ for the different Metropolis algorithms.

To obtain the exponents, consider the following steps. First, in simulations that start from the ordered state $m_{0}=1$ and $L=512$, calculate the slope $\beta / \nu z$ of the linear fit of $\ln \langle M(t)\rangle$ as a function of $\ln t$. The error bars are obtained by running simulations for $N_{\text {bin }}=5$, calculating $\langle M(t)\rangle$ for each seed, with $N_{\text {run }}=400$ runs.

Once we have calculated $\beta / \nu z$, we estimate $z$ taking the slope in a $\log -\log$ plot of $\ln F_{2}$ versus $\ln t$. We used $N_{s}=3000$ different runs starting from random spin configurations with $m_{0}=0$ for time series $\left\langle M(t)^{2}\right\rangle \times t$ and the same number of runs for time series $\langle M(t)\rangle \times t$ starting from $m_{0}=1$ (ordered state). Similarly, we repeated the numerical experiment for $N_{\text {bin }}=5$ different seeds to obtain the uncertainties. In twodimensional systems, the slope is $\phi=2 / z$ [see Eq. (4)] and so $z$ is calculated according to $\widehat{z}=2 / \widehat{\phi}$ and the uncertainty in $z$ is obtained by relation $\sigma_{z}=\left(2 / \phi^{2}\right) \sigma_{\phi}$. Here the $\widehat{\bullet}$ denotes the amount estimated from $N_{\text {bin }}=5$ different seeds. Once $z$ is calculated, the exponent $\eta=2 \beta / v$ is calculated according to $\eta=2(\widehat{\beta / \nu} z) \cdot \widehat{z}$, where $(\widehat{\beta / \nu} z)$ was estimated via magnetization decay and $\widehat{z}$ from cumulant $F_{2}$. The exponent $\theta$ was similarly obtained performing $N_{s}=3000$ different runs to evolve the time series of correlation $C(t)$ and estimating directly the slope in this case.

Tables III and V show results for the critical exponents obtained with the two algorithms. We do not observe a monotonic behavior of the critical exponents as function of $q$ in either case, but on the other hand for both cases we cannot assert, for example, that $z \in[2.09,2.15]$ (Metropolis I) and $z \in[1.97,2.20]$ (Metropolis II) or even that other exponents do not change for $q<1$, which implies that we cannot simply extrapolate the critical properties from $q=1$ to $q<1$.

\section{CONCLUSIONS}

In the nonextensive thermostatistics context, we have proposed a generalized master equation leading to a generalized Metropolis algorithm. This algorithm is local and satisfies the detailed energy balance to calculate the time evolution of spins systems. We calculate the critical temperatures using the generalized Metropolis dynamics, via equilibrium and nonequilibrium Monte Carlo simulations.

We have obtained the critical parameters performing Monte Carlo simulations in two different ways. First, we show the phase transitions from curves $\langle M\rangle$ versus $k_{B} T / J$, considering the magnetization averaging, in equilibrium, under different MC steps. Next, we use the short-time dynamics, via relaxation of magnetization from samples initially prepared of ordered or disordered states, i.e., time series of magnetizations and their moments averaged over initial conditions and over different runs.

We have also studied the Metropolis algorithm of Refs. [11, 12]. We show that it does not preserve locality or the detailed energy balance in equilibrium. While our nonequilibrium simulations corroborate results of Refs. [11,12], when we use their extension of the Metropolis algorithm (Metropolis I), the exponents and critical temperatures obtained are very different from those of our prescription (Metropolis II). When the extensive case is considered, both methods lead to the same expected values.

Simultaneously, we have developed a methodology to refine the determination of the best critical temperature. This procedure is based on optimization of the power laws of the magnetization function that relaxes from the ordered state on a logarithmic scale, via maximization of the determination coefficient of the linear fits. This approach can be extended for other spin systems, owing to its general usefulness.

For a more complete elucidation of the existence of phase transitions for $q \neq 1$, we have performed simulations for small system MC simulations, recalculating the whole lattice energy in each simple spin flip, according to the Metropolis I algorithm, only to check the variations of the critical behavior of the model. Notice that this does not apply to the Metropolis II algorithm, since it has been designed to work as the standard Metropolis one. Our numerical results show discontinuities in the magnetization, but no finite-size scaling, corroborating the results of Ref. [13], which used the broad histogram technique to show that no phase transition occurs for $q \neq 1$ using Metropolis I algorithm.

It is important to mention that only Metropolis I $[11,12]$ shows inconsistence of critical phenomena of the model since global and local simulation schemes lead to different critical properties. Metropolis II overcomes this problem since local and global prescriptions are the same even for $q \neq 1$. The broad histogram method works with a nonbiased random walk that explore the configuration space, leading to a phase transition suppression for $q \neq 1$ [13]. Nevertheless this algorithm must also be adapted to deal with the generalized Boltzmann weight in the same way the master equation needed to be modified. This is out of the scope of the present paper but this issue will be treated in the near future.

\section{ACKNOWLEDGMENTS}

The authors are partly supported by the Brazilian Research Council CNPq under Grants No. 308750/2009-8, No. 476683/2011-4, No. 305738/2010-0, and No. 476722/2010-1. The authors also thanks Professor U. Hansmann for carefully 
reading this manuscript, as well as CESUP (Super Computer Center of Federal University of Rio Grande do Sul) and Professor Leonardo G. Brunet (IF-UFRGS) for the available computational resources and support of Clustered Computing (ada.if.ufrgs.br). Finally we would like to thank the referees for their reviews.
[1] C. Tsallis, J. Stat. Phys. 52, 479 (1988).

[2] C. Tsallis and D. A. Stariolo, Physica A 233, 395 (1996).

[3] U. H. E. Hansmann, Physica A 242, 250 (1997).

[4] C. Anteneodo, C. Tsallis, and A. S. Martinez, Europhys. Lett. 59, 635 (2002).

[5] N. Destefano and A. S. Martinez, Physica A 390, 1763 (2011).

[6] A. S. Martinez, R. S. González, and C. A. S. Terçariol, Physica A 387, 5679 (2008).

[7] A. S. Martinez, R. S. González, and A. L. Espíndola, Physica A 388, 2922 (2009).

[8] Brenno Caetano Troca Cabella, A. S. Martinez, and F. Ribeiro, Phys. Rev. E 83, 061902 (2011).

[9] B. C. T. Cabella, F. Ribeiro, and A. S. Martinez, Physica A 391, 1281 (2012).

[10] R. da Silva, F. Kalil, A. S. Martinez, and J. P. M. de Oliveira, Physica A 391, 2119 (2012).

[11] N. Crokidakis, D. O. Soares-Pinto, M. S. Reis, A. M. Souza, R. S. Sarthour, and I. S. Oliveira, Phys. Rev. E 80, 051101 (2009).

[12] A. Boer, Physica A 390, 4203 (2011).

[13] J. Lima, J. S. Sá Martins, and T. J. P. Penna, Physica A 268, 553 (1999)

[14] B. Zheng, Int. J. Mod. Phys. B 12, 1419 (1998); E. V. Albano, M. A. Bab, G. Baglietto, R. A. Borzi1, T. S. Grigera, E. S. Loscar, D. E. Rodriguez, M. L. Rubio Puzzo, and G. P. Saracco, Rep. Prog. Phys. 74, 026501 (2011).

[15] H. K. Janssen, B. Schaub, and B. Z. Schmittmann, Phys. B 73, 539 (1989).

[16] D. A. Huse, Phys. Rev. B 40, 304 (1989).

[17] E. Arashiro and J. R. Drugowich de Felício, Phys. Rev. E 67, 046123 (2003).

[18] R. da Silva, N. A. Alves, and J. R. Drugowich de Felício, Phys. Lett. A 298, 325 (2002).
[19] R. da Silva and J. R. Drugowich de Felício, Phys. Lett. A 333, 277 (2004).

[20] C. S. Simões and J. R. Drugowich de Felício, Mod. Phys. Lett. B 15, 487 (2001).

[21] T. Tome and J. R. Drugowich de Felício, Mod. Phys. Lett. B 12, 873 (1998).

[22] R. da Silva and N. Alves Jr., Physica A 350, 263 (2005).

[23] R. da Silva, R. Dickman, and J. R. Drugowich de Felício, Phys. Rev. E 70, 067701 (2004).

[24] R. da Silva, N. A. Alves, and J. R. Drugowich de Felício, Phys. Rev. E 66, 026130 (2002); 67, 057102 (2003).

[25] H. A. Fernandes, R. da Silva, and J. R. Drugowich de Felicio, J. Stat. Mech. Theor. Exp. Phys. 10, P10002 (2006).

[26] E. Arashiro, J. R. Drugowich de Felício, and U. H. E. Hansmann, J. Chem. Phys. 126, 045107 (2007).

[27] E. Arashiro, J. R. Drugowich de Felício, and U. H. E. Hansmann, Phys. Rev. E 73, 040902 (2006).

[28] M. L. Rubio Puzzo and E. V. Albano, Phys. Rev. E 81, 051116 (2010).

[29] C. Tsallis, Quimica Nova 17, 468 (1994).

[30] T. J. Arruda, R. S. González, C. A. S. Terçariol, and A. S. Martinez, Phys. Lett. A 372, 2578 (2008).

[31] N. Cressie, T. R. C. Read, and J. Roy, Statist. Soc. Ser. B 46, 440 (1984).

[32] L. Nivanen, A. Le Méhauté, and Q. A. Wang, Rep. Math. Phys. 52, 437 (2003).

[33] E. P. Borges, Physica A 340, 95 (2004).

[34] M. E. J. Newman and G. T. Barkema, Monte Carlo Method in Statistical Physics (Oxford University Press, Oxford, 1999).

[35] T. Tomé and M. J. de Oliveira, Phys. Rev. E 58, 4242 (1998). 